\title{
Delta excitation in dp-elastic scattering at intermediate energies
}

\author{
Nadezhda Ladygina ${ }^{1, \star}$ \\ ${ }^{1}$ Joint Institute for Nuclear Research
}

\begin{abstract}
In this report the deuteron-proton elastic scattering reaction is studied in the range of the deuteron kinetic energy from $500 \mathrm{MeV}$ to $1300 \mathrm{MeV}$. dp-elastic scattering is considered in the relativistic multiple scattering expansion framework since the Faddeev calculation technique is not applicable at these energies. We start from the AGSequations and iterate them up to a second-order of the nucleon-nucleon interaction. The four contributions are included into consideration: one-nucleon exchange (ONE), single scattering, double scattering, and the term corresponding the delta excitation in the intermediate state. The calculations are performed for four deuteron energies: 500, 880, 1200 , and $1300 \mathrm{MeV}$. The results are presented in comparison with the experimental data on the dp-elastic scattering cross section.
\end{abstract}

\section{Introduction}

Elastic deuteron-proton scattering is the simplest example of the hadron nucleus collision, because a deuteron is the simplest nucleus containing only one proton and one neutron. In the previous papers [1],[2] we considered this reaction in the multiple scattering expansion formalism. Three contributions, one-nucleon-exchange (ONE), single-scattering (SS), and double scattering (DS), were taken into account. We got a reasonable agreement for the differential cross sections between the theoretical predictions and the experimental data almost in a whole angular range. However, the rise of the differential cross sections at the scattering angle larger than $140^{\circ}$ was not described in the approach [1],[2].

In 1969 A.Kerman and L.Kisslinger supposed that resonances can play an important role in deuteron-proton backward elastic scattering [3]. Later the double-scattering diagram with $\Delta$-isobar in the intermediate state was taken into account in dp backward scattering. The significant contribution of this diagram to the reaction amplitude was demonstrated in [4]-[6], [7]. However, the double scattering with nucleon in an intermediate state was not considered in these papers. Perhaps, it was the reason why the description of the differential cross sections energy dependence was not good enough.

The effort to take the $\Delta$-isobar into account in order to describe dp-elastic scattering was also done in [8], [9]. In these papers deuteron-proton scattering was considered in a whole angular range, not only at $\theta^{*}=180^{\circ}$. Unfortunately, the process was studied at low energies, $T_{d}<200 \mathrm{MeV}$, where the $\Delta$-isobar excitation effects are negligible.

In this paper we keep on considering deuteron-proton elastic scattering in the framework offered in papers [1],[2]. Here we add the $\Delta$-isobar contribution in our approach. The detail of the model can be found in [10]. In this paper we present only basic points and obtained results.

\footnotetext{
^e-mail: nladygina@jinr.ru
} 


\section{General formalism}

According to the three-body collision theory, the amplitude of deuteron-proton elastic scattering $\mathcal{J}$ is defined by the matrix element of the transition operator $U_{11}$ :

$$
U_{d p \rightarrow d p}=\delta\left(E_{d}+E_{p}-E_{d}^{\prime}-E_{p}^{\prime}\right) \mathcal{J}=<1(23)\left|\left[1-P_{12}-P_{13}\right] U_{11}\right| 1(23)>.
$$

Here the state $\mid 1(23)>$ corresponds to the configuration, when nucleons 2 and 3 form the deuteron state and nucleon 1 is free. Appearance of the permutation operators for two nucleons $P_{i j}$ reflects the fact that the initial and final states are antisymmetric due to an exchange of the two particles.

Following the Alt-Grassberger-Sandhas formalism [11],[12] we write the transition operator $U_{\beta \alpha}$ as:

$$
U_{\beta \alpha}=-\left(1-\delta_{\beta \alpha}\right)\left(H_{0}-z\right)-\sum_{\delta \neq \alpha} U_{\beta \delta} g_{0} t_{\delta}
$$

This transition operator depends on the potential through the channel transition-operator $t_{\alpha}$ of the two-particle subsystems:

$$
t_{\alpha}=V_{\alpha}-V_{\alpha} g_{\alpha} V_{\alpha} \quad t_{\alpha}=V_{\alpha}-V_{\alpha} g_{0} t_{\alpha},
$$

where $g_{0}$ is a free three-particle propagator and $g_{\alpha}$ is a two-particle resolvent in the three-particle space.

Iterating (2) up to the second order terms of $t_{i}$ we can present the reaction amplitude as a sum of the following four contributions: one nucleon exchange, single scattering and double scattering with the nucleon and delta in the intermediate state, -

$$
\mathcal{J}_{d p \rightarrow d p}=\mathcal{J}_{\text {ONE }}+\mathcal{J}_{S S}+\mathcal{J}_{D S}+\mathcal{J}_{\Delta}
$$

Schematically this sequence can be presented by the four diagrams figure (1). The terms in the $d p$-elastic scattering amplitude $\mathcal{J}$ in (3) correspond to: the one-nucleon-exchange (ONE) figure (1a), the single nucleon-nucleon scattering (SS) figure (1b), the double nucleon-nucleon scattering (DS) figure (1c), and scattering with $\Delta$-isobar in intermedite state figure (1d).

\section{Results and discussions.}

We applied the presented above model to describe the differential cross sections. The formal definition of the total cross section of deuteron-proton elastic scattering is given by

$$
\begin{aligned}
\sigma(d p \rightarrow d p)=(2 \pi)^{4} \frac{1}{6} \quad & \int \frac{d \vec{P}_{d}^{\prime}}{E_{d}^{\prime}} \frac{d \vec{p}^{\prime}}{E_{p}^{\prime}} \delta\left(E_{d}+E_{p}-E_{d}^{\prime}-E_{p}^{\prime}\right) \delta\left(\vec{P}_{d}+\vec{p}-\vec{P}_{d}^{\prime}-\vec{p}^{\prime}\right) . \\
& \frac{|T|^{2}}{\sqrt{\left(P_{d} p\right)^{2}-P_{d}^{2} p^{2}}},
\end{aligned}
$$

where $|T|^{2}$ is the squared invariant amplitude defined as

$$
|T|^{2}=\left|\sqrt{E_{d}^{\prime} E_{p}^{\prime}} \mathcal{J}_{d p \rightarrow d p} \sqrt{E_{d} E_{p}}\right|^{2} \equiv i n v .
$$

The expression for the differential cross section in the center-of-mass can be obtained through the scattering amplitude in the Breit frame as:

$$
\frac{d \sigma}{d \cos \theta^{*}}=\frac{(2 \pi)^{5}}{24 s}\left(s-m_{N}^{2}-M_{d}^{2}+\frac{t}{2}\right)^{2}\left|\mathcal{J}_{d p \rightarrow d p}^{(B r e i t)}(s, t)\right|^{2}
$$




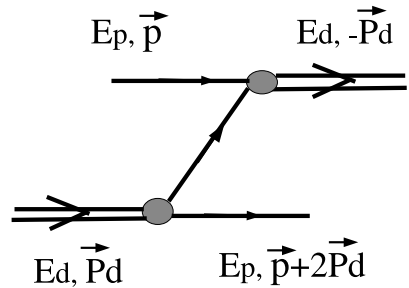

(a)

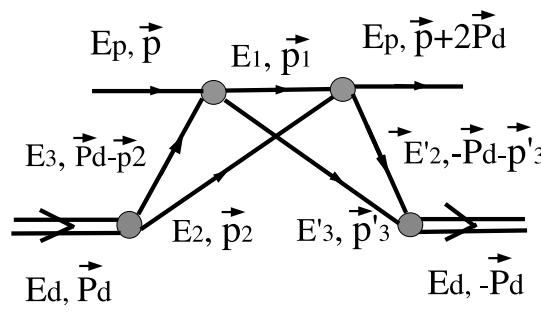

(c)

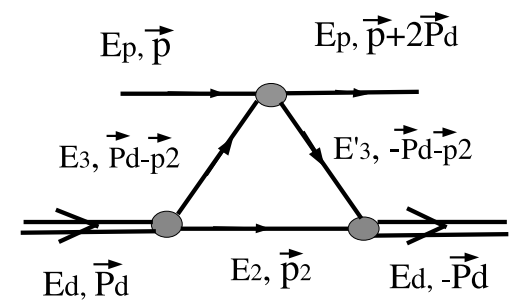

(b)

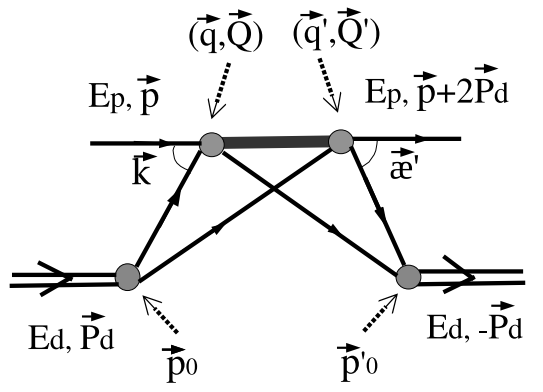

(d)

Figure 1. The diagrams included into consideration: (a) the one-nucleon exchange diagram; (b) the single scattering diagram; (c) the double scattering diagram with a nucleon in the intermediate state; (d) the double scattering diagram with $\Delta$-isobar in the intermediate state.

where $s=\left(P_{d}+p\right)^{2}$ and $t=\left(p-p^{\prime}\right)^{2}$ are invariant Mandelstam variables.

We evaluated the dp-elastic differential cross section at four energies in a wide range. The results are presented for three cases: 1) only one-nucleon exchange (ONE) and single-scattering (SS) diagrams taken into account, 2) the double-scattering (DS) term is added into the consideration, and 3) the $\Delta$-isobar excitation is included.

At first, the deuteron kinetic energy equal to $500 \mathrm{MeV}$ was considered (figure 2). One can see, the SS-diagram plays an important role up to the scattering angle equal to about $60^{\circ}$. The DS-contribution is significant at the scattering angle between $60^{\circ}$ and $160^{\circ}$. The difference between the results taking into account the double scattering and without it reaches 2-3 times at $\theta^{*}=120^{\circ}$.

This energy is not high enough for the $\Delta$-isobar manifestation. Therefore, the inclusion of the $\Delta$ contribution term into the scattering amplitude does not have to influence remarkably to the result. In fact, the results obtained with and without the $\Delta$-isobar term are indistinguishable in a whole angular range.

The second energy is equal to $880 \mathrm{MeV}$ (figure 3). One can see, the contribution of the doublescattering is very significant at this energy. The inclusion of the DS-term into consideration allows to describe the behaviour of the differential cross section at the scattering angle range $60^{\circ}-140^{\circ}$. The difference between ONE+SS curve and ONE+SS+DS one reaches about 15 times. Note, the $\Delta$ excitation begins to manifest itself at the angle equal to about $120^{\circ}$ and describes the behaviour of the experimental data at the angle above $140^{\circ}$ where the differential cross section sharply increases. 


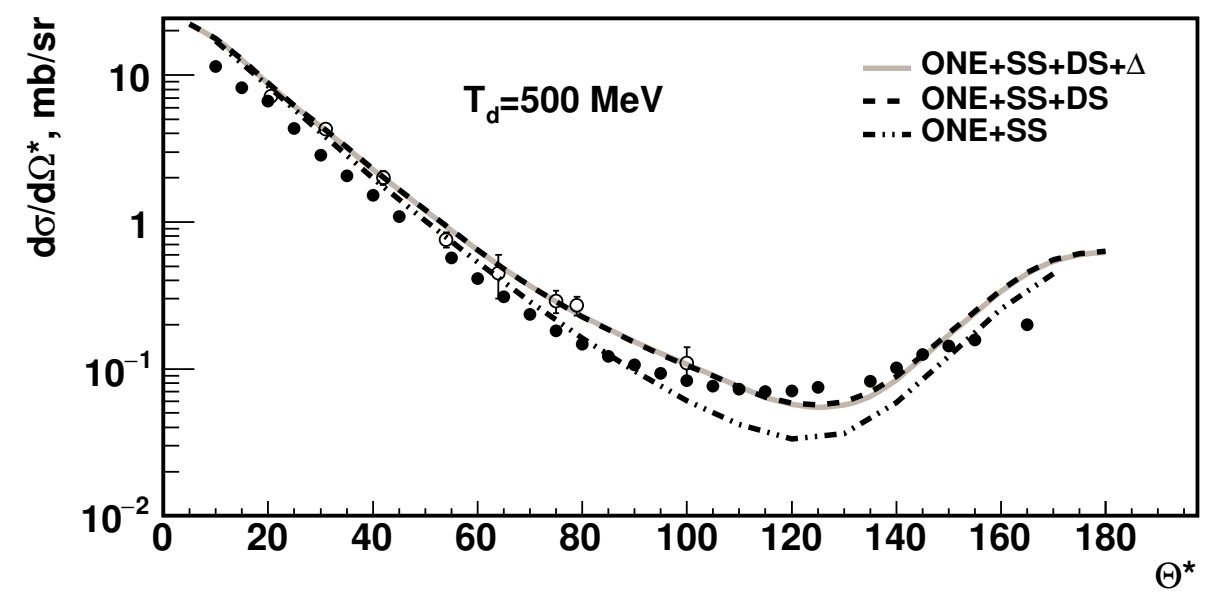

Figure 2. The differential cross section at the deuteron kinetic energy of $500 \mathrm{MeV}$ as a function of the c.m. scattering angle. The data are taken from [13] (०), and [14] $(\bullet)$.

The dp-elastic differential cross sections at energies equal to $T_{d}=1200 \mathrm{MeV}$ and $T_{d}=1300$ $\mathrm{MeV}$ are presented in figure 4 and figure 5, respectively. Here we can observe further enhancement of the double scattering term influence on the result. Also the contribution of the $\Delta$ - isobar increases significantly at the scattering angle above $140^{\circ}$.

Analysing the obtained results one can conclude the following. The differential cross sections are described quite well up to the scattering angles equal to $60^{\circ}$ taking into account only the one-nucleonexchange and single-scattering terms. If we consider the dp-elastic scattering at the angles large than $60^{\circ}$, it is necessary to include the double scattering term into consideration. It should be noted the double-scattering contribution into the reaction amplitude increases with the deuteron energy growing and may change the value of the differential cross section on a few orders in comparison with the result obtained without inclusion of the DS-term.

The sharp rise of the differential cross section at $\theta^{*} \geq 140^{\circ}$, which is observed in the experimental data, points at the appearance of the addition reaction mechanism. The inclusion of the $\Delta$-excitation term into consideration allowed to describe this rise of the differential cross section at the large scattering angles. The contribution of the $\Delta$-isobar mechanism grows with the initial deuteron energy. It is negligible at $T_{d}=500 \mathrm{MeV}$ and significant at $T_{d}=1300 \mathrm{MeV}$.

In such a way we got a good description of the differential cross section of deuteron-proton elastic scattering in a whole angular range, from $0^{\circ}$ to $180^{\circ}$ of the scattering angle, in a wide deuteron energy interval, between $500 \mathrm{MeV}$ and $1300 \mathrm{MeV}$.

Acknowledgements: The author is grateful to Dr. V.P. Ladygin for fruitful discussions and interest in this problem. This work has been supported by the Russian Foundation for Basic Research under grant $N^{0}$ No.16-02-00203a. 


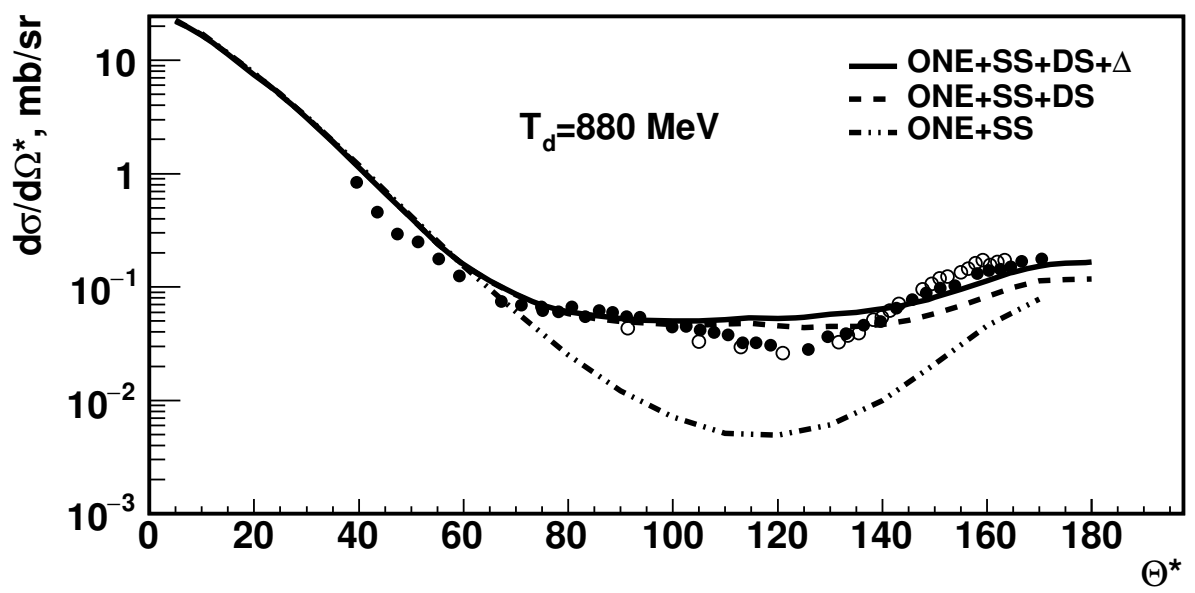

Figure 3. The differential cross section at the deuteron kinetic energy of $880 \mathrm{MeV}$ as a function of the c.m. scattering angle. The data are taken from [15] (•), and [16] (०).

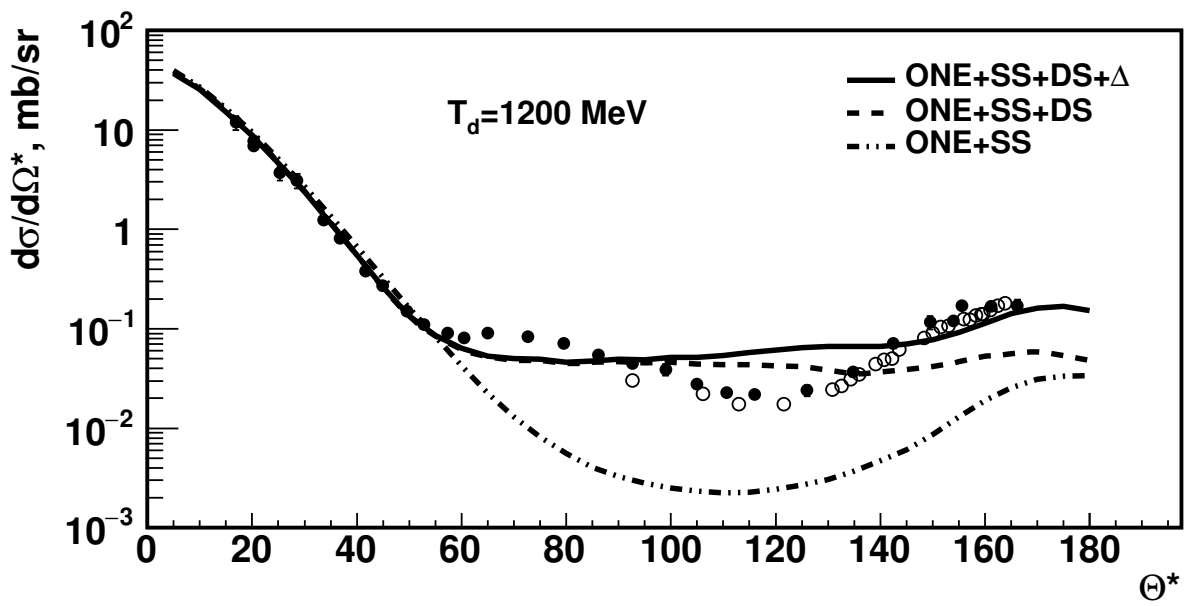

Figure 4. The differential cross section at the deuteron kinetic energy of $1200 \mathrm{MeV}$ as a function of the c.m. scattering angle. The data are taken from [16] (०), and [17] (•).

\section{References}

[1] N.B. Ladygina, Phys. At. Nucl. 71, 2039 (2008)

[2] N.B. Ladygina, Eur. J. Phys. A 42, 91 (2009)

[3] A.K. Kerman, L.S. Kisslinger, Phys. Rev. 180, 1483 (1969)

[4] L.A. Kondratyuk, F.M. Lev, Sov. J. Nucl. Phys. 26, 153 (1977) 


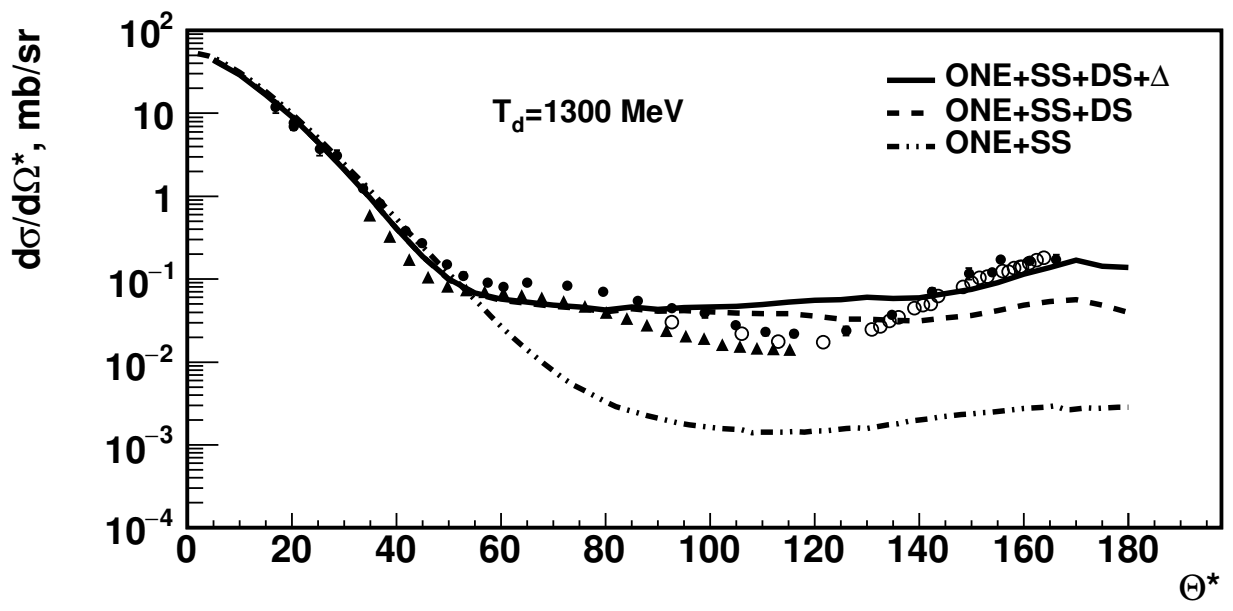

Figure 5. The differential cross section at the deuteron kinetic energy of $1300 \mathrm{MeV}$ as a function of the c.m. scattering angle. The data are taken for $T_{d}=1200 \mathrm{MeV}$ from $T_{d}=1180 \mathrm{MeV}[16]$ (०), [17]( $\bullet$ ), and $T_{d}=1282$ $\mathrm{MeV}[18](\mathbf{\Lambda})$.

[5] L.A. Kondratyuk, F.M. Lev, L.V. Shevchenko, Sov. J. Nucl. Phys. 29, 558 (1979)

[6] L.A. Kondratyuk, F.M. Lev, L.V. Shevchenko, Yad. Fiz. 33, 1208 (1981)

[7] Yu.N. Uzikov, Fiz.Elem.Chast.Atom.Yadra 29, 1405 (1998)

[8] A. Deltuva, K. Chmielewski, P.U. Sauer, Phys. Rev. C 67, 034001 (2003)

[9] A. Deltuva, R. Machleidt, P.U. Sauer, Phys. Rev. C 68, 024005 (2003)

[10] N.B.Ladygina, Eur. J. Phys. A 52, 199 (2016)

[11] E.O. Alt, P. Grassberger, W. Sandhas, Nucl. Phys. B 2, 167 (1967)

[12] E. Schmid, H. Ziegelmann, The Quantum Mechanical Three-Body Problem (Oxford, Pergamon Press, 1974)

[13] R.D. Shamberger, Phys. Rev. 85, 424 (1952)

[14] K. Hatanaka et al., Phys. Rev. C 66, 044002 (2002)

[15] N.E. Booth et al., Phys. Rev. D 4, 1261 (1971)

[16] J.C. Alder et al., Phys. Rev. C 6, 2010 (1972)

[17] E.T. Boschitz et al., Phys. Rev. C 6, 457 (1972)

[18] E. Gülmez et al., Phys. Rev. C 42, 2067 (1991) 